\title{
FURTHER EVOLUTION OF V 1016 CYGNI, 1974-77
}

\author{
F. Ciatti, A. Mammano, A. Vittone \\ Asiago Astrophysical Observatory
}

SUMMARY :

$\checkmark 1016$ Cygni is a peculiar variable star, whose brightness increased from about 16 mag in 1963 (previously it was known to have a LPV spectrum) to about $11 \mathrm{mag}$ in 1967. Since then it has been nearly constant at maximum. Our last photo metric observations, from May to August 1977, indicate that the star is still in its protracted maximum at a constant brightness. We have measured $B=11.1$ and $V=10.8$ ( $\pm 0.2 \mathrm{mag}$ s.e.).

The spectrum is very rich of nebular forbidden lines, resembling that of a planetary nebula with very high density. Its evolution has shown a steady increa se of the excitation stage, while low-excitation lines, like [FeII] and 0I 8446 A, were still present. The spectrograms obtained in 1976 and 1977 in the wavelength range 3580-10900 A, show a further increase of the degree of excitation, as deduc ed from the strengthening of the following emissions relative to nearby 1 ines: HeII (I.P.54 eV) 4199, 4541, 4686 A

[ArV] (I.P.60 eV) 6435,7006 [CaVI] (I.P.84 eV) 5460 [FeVII] (I.P.109 eV) 3587, 3758, 4893, 4944, 5721, 6085

The properties of $V 1016 \mathrm{Cyg}$ can be explained with a double star model (Mam mano and Ciatti, Astron.Astrophys.39,405;1975). The long period variable, still indicated from $T i O$ and $V O$ bands, and infrared variability with $P=450^{d}$, is eject ing mass at a rate of $10^{-5} \mathrm{Me} /$ year. The resulting nebula is excited by a very hot companion, which is responsible for the outburst. An evolution toward some kind of compact planetary nebula is suggested. 